\title{
Metabolic and carbohydrate characteristics of different phenotypes of polycystic ovary syndrome
}

\author{
Ebru Çelik ${ }^{1}$, Ilgın Türkçüoğlu ${ }^{1}$, Banış Ata ${ }^{2}$, Abdullah Karaer ${ }^{1}$, Pınar KınııI ${ }^{1}$, Sevil Eraslan ${ }^{1}$, Çağatay Taşkapan ${ }^{3}$, Bülent Berker ${ }^{4}$ \\ 'Department of Obstetrics and Gynecology, İnönü University School of Medicine, Malatya, Turkey \\ ${ }^{2}$ Department of Obstetrics and Gynecology, Koç University School of Medicine, Istanbul, Turkey \\ ${ }^{3}$ Department of Clinical Biochemistry, İnönü University School of Medicine, Malatya, Turkey \\ ${ }^{4}$ Department of Obstetrics and Gynecology, Ankara University School of Medicine, Ankara, Turkey
}

\begin{abstract}
Objective: To compare the prevalence of various metabolic and cardiovascular risk factors and insulin resistance between polycystic ovary syndrome (PCOS) patients with or without hyperandrogenism.

Material and Methods: This is a retrospective cross-sectional study involving women with PCOS as diagnosed according to the Androgen Excess (AE) Society definition $(n=504)$ and women with normoandrogenemic PCOS $(n=183)$. Anthropometrics, lipid profile, glucose, insulin, oral glucose tolerance test (OGTT), and reproductive hormone levels were evaluated.

Results: Women with PCOS diagnosed according to the AE Society had a significantly higher prevalence of metabolic syndrome compared with the normoandrogenemic PCOS phenotype: odds ratio (OR) 2.95 [95\% confidence interval (CI) 1.21-7.21]. There was no significant difference in the prevalence glucose intolerance test between the groups [OR: 2.15, 95\% CI 0.71-6.56]. The prevalence of low high density lipoprotein (HDL)-cholesterol in the group under the AE-PCOS Society criteria was higher than that of the normoandrogenemic PCOS group [OR: 2.82, 95\%CI 1.29-3.36].

Conclusion: The risks of metabolic syndrome and cardiovascular disease may vary among the phenotypes of PCOS based on the Rotterdam criteria. This new data may be of reference in informing women with PCOS, although further prospective studies are needed to validate this proposition. (J Turk Ger Gynecol Assoc 2016; 17: 201-8)
\end{abstract}

Keywords: Polycystic ovary syndrome, diagnostic categories, metabolic syndrome, hyperanderogenism, insulin resistance

Received: 1 August, 2016 Accepted: 27 October, 2016

\section{Introduction}

Polycystic ovary syndrome (PCOS) is a frequent endocrine abnormality of women in the reproductive period, and is related with infertility and menstrual irregularities $(1,2)$. PCOS is a heterogeneous condition, presenting with hyperandrogenism, polycystic ovarian morphology (POM), and oligomenorrhea (3-5).

The etiology of PCOS is elusive, but its long-term implications, including the risk of cardiovascular disease, type-2 diabetes mellitus, and endometrial carcinoma, are well known (6-8). However, PCOS is regarded as the most common endocrine abnormality of women in the reproductive period; its predicted frequencies vary in the literature, ranging from $2.2 \%$ to up to $26 \%(2,9-12)$. A major reason for this variation is the absence of a consensus on the diagnostic criteria. Opinions differ on whether the presence of hyperanderogenism is an absolute requirement for a diagnosis of PCOS. The same holds true for the requirement of POM (13).

Chronologically, the National Institutes of Health (NIH) proposed the first diagnostic criteria. The NIH criteria required the combination of chronic oligo/anovulation and clinical or biochemical evidence of hyperandrogenism, with the exclusion of related disease (14). Subsequently, the Rotterdam European Society of Human Reproduction and the Embryology/American Society for Reproduction Medicine (ESHRE/ASRM) Sponsored PCOS (Rott-PCOS) Consensus Workshop group proposed the addition of POM to the NIH criteria, with a statement that PCOS could be diagnosed when any two of these three criteria were present (13). More recently, the Androgen Excess Society (AES) proposed new diagnostic criteria and stated that androgen excess is the sine qua non of PCOS and the syndrome must only be diagnosed in the presence of hyperanderogenism in combination with oligo/anovulation and/or POM (15). Compared to the recent AES criteria, the Rotterdam criteria include an additional PCOS phenotype, comprising women with POM and oligo/anovulation in the absence of androgen excess. It is still unknown whether this new phenotype is also related to long-term health risks, such as metabolic syndrome, insulin resistance, and obesity $(13,16)$. Diagnosing normoandrogenemic women with PCOS has attracted 
criticism (15). Although the normoandrogenemic PCOS phenotype appears to be weakly associated with adverse reproductive outcomes and metabolic syndrome compared with hyperandrogenemic PCOS, some evidence suggests that women with normoandrogenemic PCOS present with more severe insulin resistance (IR) and dyslipidemia (17-19).

Whether women with only POM and oligo/amenorrhea bear similar metabolic and cardiovascular risks as compared with hyperandrogenemic women diagnosed with PCOS as per the AES criteria remains to be determined. This study aims to compare the prevalence of various metabolic and cardiovascular risk factors and insulin resistance between women diagnosed as PCOS with or without hyperandrogenism.

\section{Material and Methods}

This is a retrospective cohort study involving women who presented to our Gynecology Clinic between April 2011 and August 2012. The study protocol was approved by the Institutional Ethic Committee.
Demographic features and medical information regarding menstrual cycles, obstetric and gynecological history, and previous medication and/or disease were collected from the medical records. Body mass index (BMI) was calculated as body weight (in kilograms) divided by height (in meters squared). The waist to hip ratio $(\mathrm{W} / \mathrm{H})$ was calculated from dividing the waist circumference by the hip circumference.

A total of 1048 consecutive women diagnosed with PCOS were analyzed. PCOS was diagnosed with regard to the Rotterdam criteria, i.e., according to the existence of at least two of the following three features: 1) oligo- or amenorrhea; 2) clinical or biochemical hyperandrogenism; or 3) POM on pelvic ultrasound, after exclusion of other causes of hyperandrogenism, thyroid disorders, hyperprolactinemia, Cushing syndrome, and late onset congenital adrenal hyperplasia. Women with type1 diabetes mellitus, hepatic or renal pathology, or receiving medication, which could affect carbohydrate metabolism or lipid profile, were also excluded. The follow-chart of subjects is presented in Figure 1.

8906 cases from the database of women who attended to gynecological outpatient

Excluded $(n=665)$; missing history of cycle length $(n=86)$; lack of ultrasonography (103); missing information on hirsutism $(n=115)$; missing data on thyroid and/or prolactin levels $(n=361)$
Excluded $(n=830)$; chronic disease $(n=165)$; congenital uterine and/or ovarian dysplasia $(n=27)$; endometriosis $(n=106)$; hyperprolactinemia $(n=88)$; abnormal thyroid function $(n=322)$; diabetes mellitus type-1 $(n=4)$; premature ovarian failure

$(n=41)$; taking medications that may affect the carbonhydrate metabolism, lipid profile or endocrine parameters $(n=77)$

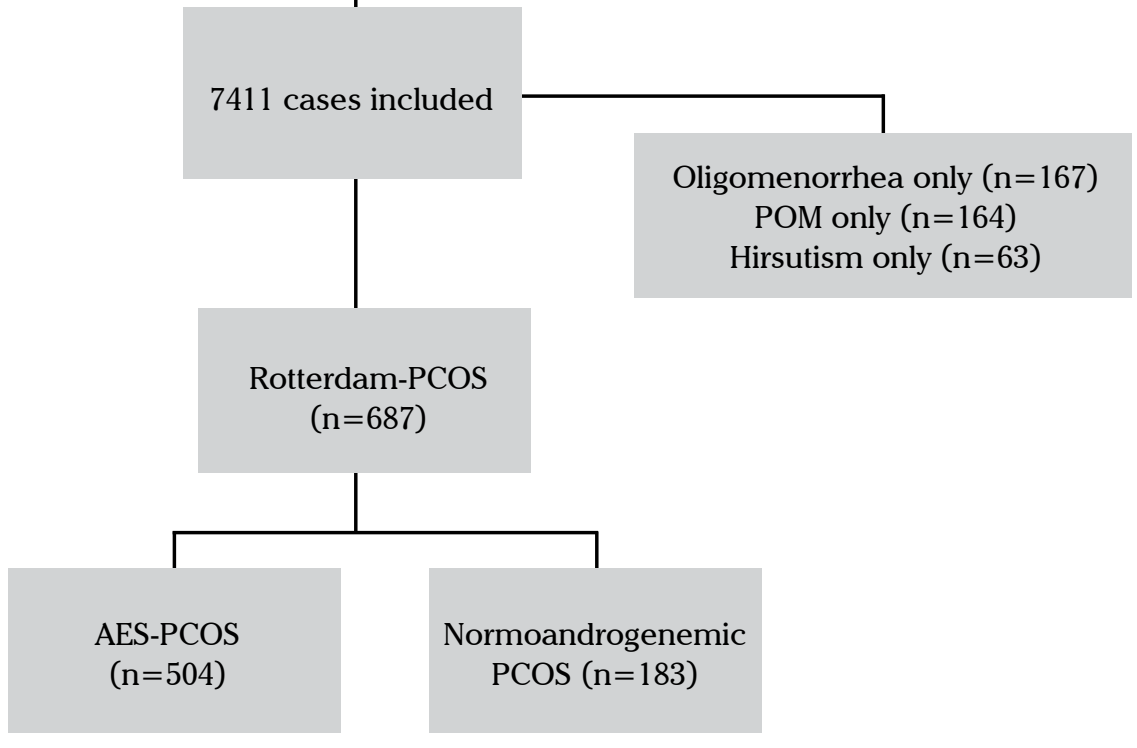

Figure 1. The flowchart of subjects who attended the clinic 


\section{PCOS subtypes}

The participants were divided into two groups: i) those fulfilling the AES criteria ( $n=504)$ (15) and ii) women with normoandrogenemic PCOS (oligomernorrhea with POM; $n=183$ ).

The criteria used to determine the components of the PCOS phenotypes were:

- Clinical hyperandrogenism was determined with a modified Ferriman-Gallewey score $>8$ (20).

- Biochemical hyperandrogenism was defined with the existence of at least one of the following findings: serum total testosterone level $(\mathrm{tT})>65.82 \mathrm{ng} / \mathrm{dL}$, serum dehydroepiandrosterone sulfate (DHEAS) level $>374.9 \mu \mathrm{g} / \mathrm{dL}$ and free androgen index (FAI) $>4.94$ (calculated on the basis of the $95^{\text {th }}$ percentile of basal serum androgen normality in the control group of 70 healthy, non-hirsute, eumenorrheic women from the same area (unpublished data).

- Oligomenorrhea was defined as having $<8$ menstrual cycles/year or menstrual cycles for more than 35 days.

- POM was defined with an antral follicle count (2-9 mm) of $\geq 12$ in at least one ovary.

\section{Definition of insulin resistance}

Insulin resistance was predicted by using the homeostatic model assessment of insulin resistance (HOMA-IR) (21). The following formula was used to calculate HOMA-IR: (fasting insulin in $\mu \mathrm{U} / \mathrm{mL} \mathrm{x}$ fasting glucose in $\mathrm{mg} / \mathrm{dL}$ )/405. A HOMA-IR value $\geq 3.8$ was considered to represent insulin resistance (22). A 120-minute 75-g oral glucose tolerance test (OGTT) was done on a random day of the cycle. Impaired glucose tolerance test (IGTT) was defined by an abnormal glucose value following the 75-g OGTT, with cut-off values between 140 and 199 for 120 minute. A value of $200 \mathrm{mg} / \mathrm{dl}$ or higher subsequent to 75 -g OGTT were considered as diabetes mellitus type-2 (23).

\section{Definition of metabolic syndrome (MetS) and dyslipidemia}

The diagnosis of MetS was made in accordance to the definitions proposed by the National Cholesterol Education Program Adult Treatment Panel III (NCEP ATP III). Based on the NCEP ATP III, the diagnosis of MetS in women was defined by the presence of at least three of the following features: (i) waist circumference $\geq 88$ $\mathrm{cm}$, (ii) serum triglyceride (TG) level $\geq 150 \mathrm{mg} / \mathrm{dL}$, (iii) serum high density lipoprotein (HDL-cholesterol) level $<50 \mathrm{mg} / \mathrm{dL}$ or the previous consumption of lipid lowering medication, (iv) blood pressure $\geq 130 / 85 \mathrm{mmHg}$ or the use of anti-hypertensive medication, (v) fasting blood glucose $\geq 100 \mathrm{mg} / \mathrm{dL}$ (24).

Dyslipidemia was diagnosed according to the definitions proposed by the Framingham/Adult Treatment Panel (ATP) III criteria (25). Regarding Framingham/ATP III, the diagnosis of dyslipidemia in women was defined by the following features: (i) low density lipoprotein (LDL-cholesterol) $\geq 130 \mathrm{mg} / \mathrm{dL}$, (ii) HDL-cholesterol $<50 \mathrm{mg} / \mathrm{dL}$, (iii) triglyceride $\geq 150 \mathrm{mg} / \mathrm{dL}$, (iv) total cholesterol (TC) $\geq 200 \mathrm{mg} / \mathrm{dL}$, (v) TC/HDL $\geq 5.6$.

\section{Laboratory analyses}

Comprehensive endocrine screening, including early follicular phase follicle stimulating hormone (FSH), luteinizing hormone $(\mathrm{LH})$, total testosterone (tT), dehydroepiandrosterone sulfate
(DHEAS), sex hormone-binding globulin (SHBG), thyroid stimulating hormone (TSH), and prolactin, was carried out. Plasma insulin, FSH, LH, SHBG, DHEAS, and TSH levels were analyzed by a chemiluminescence method (Immulite 2000, Siemens Medical Solutions Diagnostics; Los Angeles, CA, USA). FAI was determined as follows: $\mathrm{FAI}=\mathrm{tT} \times 100 / \mathrm{SHBG}$.

Fasting glucose and insulin, lipids, and carbohydrates levels were also determined. The plasma glucose, total cholesterol, HDL-cholesterol, and TG levels were tested by the spectrophotometric method (Abbott Trade C16000, Abbott Laboratories; Abbott Park, IL, USA). LDL-cholesterol and VLDL-cholesterol were calculated using Friedewald formulation. Highly sensitive C-reactive protein (hs-CRP) was analyzed by the nephelometric method (Siemens Dade-Behring BNII, Siemens Healthcare Diagnostics Inc.; Newark, BE, USA).

\section{Statistical analyses}

Continuousvariables are defined herein with the mean \pm standard deviation (SD), and categorical variables with numbers and percentages as appropriate. Baseline features of the different PCOS phenotypes were compared by independent samples $t$ test. Proportions were compared using the chi-square test. Statistical significance was set at a two sided $p$ value $\leq 0.05$. The Statistical Package for Social Sciences (SPSS) was used for statistical analysis (version 19.0, SPSS Inc.; Chicago, IL, USA).

\section{Results}

\section{Study population}

The medical records of 8906 women were reviewed; 665 women were excluded due to inadequate data, including for unavailable data on the history of the cycle length $(n=86)$, ultrasonographic examination ( $n=103)$, hirsutism ( $n=115)$, thyroid and/or prolactin laboratory assay $(\mathrm{n}=361)$. An additional 830 women were excluded due to the presence of concomitant pathology, including other relevant chronic diseases $(n=165)$, congenital uterine anomalies and/or ovarian dysplasia $(n=27)$, endometriosis $(n=106)$, hyperprolactinemia $(n=88)$, abnormal thyroid function $(n=322)$, diabetes mellitus type-1 $(n=4)$, premature ovarian failure $(n=41)$, and the previous consumption of medications that may affect the carbohydrate pathway, lipid profile, or endocrine parameters $(n=77)$ (Figure 1). The final study population included 687 women diagnosed with PCOS based on Rott-PCOS criteria. While 504 of these women met the AES -PCOS criteria, 183 women were classified as normoandrogenemic PCOS. The prevalence of different PCOS phenotypes is presented in Table 1.

The prevalence of women with clinical and/or biochemical hyperandrogenism, oligomenorhea (OM), and POM was 7.6, 8.5 , and $7.1 \%$, respectively.

Demographic characteristics, and the biochemical and anthropometric features of the participants are presented in Table 2. There were no differences among the PCOS subgroups regarding age $(p=0.62$; Table 2$)$. The mean concentration of C-reactive protein (CRP) in women diagnosed as PCOS according to the AES criteria was significantly higher than that of women classified as normoandrogenemic PCOS (5.96 \pm 6.92 vs. $4.21 \pm 3.12 \mathrm{mg} / \mathrm{L}, \mathrm{p}=0.003)$. 
Table 1. Prevalence of PCOS phenotypes among the same population

\begin{tabular}{|l|c|c|}
\hline Phenotypes & $\mathbf{n}$ & $\%$ \\
\hline Screened population & 7411 & \\
\hline Rotterdam Criteria - PCOS & 687 & 9.3 \\
\hline AES Criteria - PCOS & 504 & 6.8 \\
\hline PCOS with all 3 criteria (HA+POM+OM) & 277 & 3.7 \\
\hline Normoandrogenemic PCOS (OM+POM) & 183 & 2.5 \\
\hline Ovulatory PCOS (HA+POM) & 144 & 1.9 \\
\hline HA+OM & 83 & 1.1 \\
\hline $\begin{array}{l}\text { PCOS: polycystic ovary syndrome; AES: Androgen Excess Society; HA: hy- } \\
\text { perandrogenism; POM: polycystic ovary morphology; OM: oligemenorrhea }\end{array}$ \\
\hline
\end{tabular}

\section{Prevalence of insulin resistance}

A comparison of the data between AES-PCOS and normoandrogenemic PCOS revealed a significant difference for the carbohydrate traits assessed, including HOMA-IR $(\mathrm{p}=0.004)$, fasting insulin (0.007), and fasting glucose $(\mathrm{p}=0.001)$.

The overall prevalence of elevated fasting blood glucose and insulin resistance among the PCOS patients was $17.8 \%$ and $31.7 \%$, respectively. While the rates of elevated blood glucose and insulin resistance were $19.9 \%$ and $32.8 \%$ in the AES-PCOS group, within the women classified as normoandrogenemic PCOS, these rates were $10.0 \%$ and $26.3 \%$, respectively $(p=0.01$ and 0.34 , respectively). The respective odds ratios for insulin resistance in patients diagnosed as PCOS according to the AES criteria was 1.36 [95\% confidence interval (CI), 0.77-2.58] when compared with the subjects classified as normoandrogenemic PCOS (Table 3).

Table 2. Demographic, anthropometric, and metabolic parameters of various PCOS phenotypes

\begin{tabular}{|c|c|c|c|}
\hline Variables & $\operatorname{AES}-\operatorname{PCOS}\left(\mathbf{n}_{\text {avail }}\right)$ & Normoandrogenemic PCOS $\left(n_{\text {avai }}\right)$ & $\mathbf{p}$ \\
\hline Age & $24.73 \pm 5.86(504)$ & $24.48 \pm 5.54(183)$ & 0.62 \\
\hline Body Mass Index $\left(\mathrm{kg} / \mathrm{m}^{2}\right)$ & $26.12 \pm 6.26(168)$ & $23.96 \pm 4.51(24)$ & 0.10 \\
\hline Waist circumference (mm) & $78.66 \pm 13.53(158)$ & $77.92 \pm 17.20(19)$ & 0.82 \\
\hline Hip circumference (mm) & $103.25 \pm 17.09(158)$ & $102.46 \pm 21.03(19)$ & 0.85 \\
\hline Waist to hip ratio & $0.79 \pm 0.09(158)$ & $0.76 \pm 0.10(19)$ & 0.17 \\
\hline Systolic blood pressure (mmHg) & $110(110-120)(99)$ & $115(102-120)(19)$ & 0.53 \\
\hline Diastolic blood pressure (mmHg) & $70(62-80)(99)$ & $70(60-80)(19)$ & 0.20 \\
\hline Follicule stimulating hormone (mIU/mL) & $5.67 \pm 2.09(502)$ & $5.77 \pm 2.03(183)$ & 0.55 \\
\hline Luteinizing hormone $(\mathrm{mIU} / \mathrm{mL})$ & $7.23 \pm 5.24(503)$ & $8.13 \pm 5.51(183)$ & 0.05 \\
\hline Thyroid stimulating hormone (mIU/mL) & $1.58 \pm 0.84(504)$ & $1.58 \pm 0.83(183)$ & 0.99 \\
\hline Prolactin (ng/mL) & $13.16 \pm 7.81(504)$ & $13.58 \pm 7.88(183)$ & 0.54 \\
\hline Total testosterone (ng/dL) & $48.03 \pm 89.04(346)$ & $31.84 \pm 12.46(72)$ & 0.12 \\
\hline Free testosterone (ng/dL) & $6.40 \pm 9.35(183)$ & $2.88 \pm 2.11(32)$ & $<0.01^{*}$ \\
\hline Sex hormone binding globulin (nmoL/mL) & $40.73 \pm 31.95(346)$ & $64.64 \pm 43.84(72)$ & $<0.01$ \\
\hline Free androgen index & $6.11 \pm 9.43(346)$ & $2.32 \pm 1.39(72)$ & $<0.01^{*}$ \\
\hline Dehydroepiandrosterone sulfate $(\mu \mathrm{g} / \mathrm{dL})$ & $243.75 \pm 117.05(368)$ & $181.89 \pm 79.67(80)$ & $<0.001^{*}$ \\
\hline Ferriman-Gallewey score, median (IQR) & $13(10-18)(404)$ & $6(4-7)(175)$ & $<0.001^{*}$ \\
\hline Fasting Insulin $(\mu \mathrm{IU} / \mathrm{mL})$ & $15.69 \pm 20.35(298)$ & $10.84 \pm 10.16(57)$ & $0.007^{*}$ \\
\hline Fasting glucose $(\mathrm{mg} / \mathrm{dL})$ & $91.81 \pm 12.60(442)$ & $87.83 \pm 9.83(120)$ & $0.001 *$ \\
\hline${ }^{\S} \mathrm{HOMA}-\mathrm{IR}$ & $4.53 \pm 6.65(296)$ & $2.94 \pm 2.96(57)$ & $0.004 *$ \\
\hline Total Cholesterol (mg/dL) & $170.09 \pm 35.59(382)$ & $168.60 \pm 38.22(87)$ & 0.73 \\
\hline Triglycerides (mg/dL) & $113.14 \pm 64.62(382)$ & $98.98 \pm 52.41(87)$ & $0.03^{*}$ \\
\hline 'HDL-cholesterol (mg/dL) & $48.01 \pm 11.76(383)$ & $52.28 \pm 13.15(85)$ & $0.004^{*}$ \\
\hline LDL-cholesterol (mg/dL) & $101.15 \pm 29.14(374)$ & $96.95 \pm 31.98(86)$ & 0.23 \\
\hline${ }^{\mathrm{Y} V L D L}$-cholesterol (mg/dL) & $23.29 \pm 14.88(373)$ & 19.84 \pm 11.26 (83) (Matthews, Hosker et al.) & $0.02 *$ \\
\hline Triglycerides/"HDL-cholesterol ratio & $2.63 \pm 1.92(376)$ & $2.06 \pm 1.41(85)$ & $0.002^{*}$ \\
\hline C-reactive protein $(\mathrm{mg} / \mathrm{L})$ & $5.96 \pm 6.92(253)$ & $4.21 \pm 3.12(62)$ & $0.003 *$ \\
\hline \multicolumn{4}{|c|}{$\begin{array}{l}\text { AES: Androgen Excess Society; } \mathrm{n}_{\text {avail }} \text { : the number of women available for each characteristic measured; HOMA-IR: homeostatic model assessment of } \\
\text { insulin resistance; HDL-cholesterol: high density lipoprotein; LDL-cholesterol: low density lipoprotein; VLDL-cholesterol: very low density lipoprotein } \\
\text { Data are expressed as the mean } \pm \text { SD unless otherwise was stated. } \\
\text { *Statistically significant }(\mathrm{p}<0.05) \text {. }\end{array}$} \\
\hline
\end{tabular}


Table 3. The prevalence of obesity, insulin resistance, and the components of metabolic syndrome according to the International Diabetes Federation criteria and the compositions of dyslipidemia according to the Adult Treatment Model III National Cholesterol Education Program criteria among all the PCOS phenotypes

\begin{tabular}{|c|c|c|c|c|}
\hline Variables & AES-PCOS (n) & $\begin{array}{l}\text { Normoandrogenemic } \\
\text { PCOS (n) }\end{array}$ & $\mathbf{p}^{*}$ & $\begin{array}{c}\text { Odds Ratio } \\
\text { (95\% Confidence interval) }\end{array}$ \\
\hline $\mathrm{BMI} \geq 25\left(\mathrm{~kg} / \mathrm{m}^{2}\right)$ & $49.4 \%(83 / 168)$ & $41.7 \%(10 / 24)$ & 0.48 & $0.73[0.31-1.74]$ \\
\hline $\mathrm{BMI} \geq 27\left(\mathrm{~kg} / \mathrm{m}^{2}\right)$ & $39.9 \%(67 / 168)$ & $37.5 \%(9 / 24)$ & 0.82 & $0.90[0.37-2.18]$ \\
\hline $\mathrm{BMI} \geq 30\left(\mathrm{~kg} / \mathrm{m}^{2}\right)$ & $25.6 \%(43 / 168)$ & $12.5 \%(3 / 24)$ & 0.16 & $1.59[0.63-3.97]$ \\
\hline Insulin resistance (HOMA-IR $\geq 3.8$ ) & $32.8 \%(97 / 296)$ & $26.3 \%(15 / 57)$ & 0.34 & $1.36[0.77-2.58]$ \\
\hline Oral Glucose Test Intolerance & $21.2 \%(31 / 146)$ & $11.1 \%(4 / 36)$ & 0.23 & $2.15[0.71-6.56]$ \\
\hline Diabetes Mellitus type-2 & $1.4 \%(2 / 146)$ & - & - & - \\
\hline Fasting glucose $\geq 100 \mathrm{mg} / \mathrm{dL}$ & $19.9 \%(88 / 442)$ & $10.0 \%(12 / 120)$ & $0.01 *$ & $2.23[1.17-4.24]$ \\
\hline Waist circumference $\geq 88 \mathrm{~cm}$ & $50.6 \%(80 / 158)$ & $42.1 \%(8 / 19)$ & 0.48 & $1.41[0.54-3.69]$ \\
\hline Blood pressure $\geq 130 / 85 \mathrm{mmHg}$ & $5.1 \%(5 / 99)$ & $5.3 \%(1 / 19)$ & 0.96 & $0.96[0.15-6.06]$ \\
\hline HDL-cholesterol < $<0$ mg/dL & $59.4 \%(228 / 384)$ & $41.2 \%(35 / 85)$ & $0.002^{*}$ & $2.82[1.29-3.36]$ \\
\hline Triglycerides $\geq 150 \mathrm{mg} / \mathrm{dL}$ & $23.0 \%(88 / 322)$ & $14.9 \%(13 / 87)$ & 0.09 & $1.70[0.90-3.21]$ \\
\hline Total cholesterol $\geq 200 \mathrm{mg} / \mathrm{dL}$ & $18.6 \%(71 / 382)$ & $17.2 \%(15 / 87)$ & 0.77 & $1.09[0.59-2.02]$ \\
\hline LDL-cholesterol $\geq 130 \mathrm{mg} / \mathrm{dL}$ & $15.2 \%(57 / 374)$ & $11.6 \%(10 / 86)$ & 0.39 & $1.21[0.59-2.48]$ \\
\hline Triglycerides/HDL-cholesterol $\geq 5.6$ & $8.5 \%(32 / 376)$ & $3.5 \%(3 / 85)$ & 0.12 & $2.54[0.76-8.51]$ \\
\hline $\begin{array}{l}\text { Metabolic syndrome (any more than } \\
\text { two components) }\end{array}$ & $25.4 \%(62 / 244)$ & $10.3 \%(6 / 58)$ & $0.01^{*}$ & $2.95[1.21-7.21]$ \\
\hline
\end{tabular}

The prevalence of IGTT was $19.9 \%$ in the whole PCOS population. In the AES-PCOS group, 31 of 146 patients $(21.2 \%)$ had impaired glucose tolerance during OGTT and of these, two patients had DM type $2(1.4 \%)$, while the prevalence rate for the abnormal glucose tolerance test in the normoandrogenic PCOS group was $11.1 \%(p=0.23)$. The respective odds ratio for the glucose intolerance test in patients diagnosed as PCOS according to the AES criteria was 2.15 [95\% CI, 0.71-6.56] when compared with subjects classified as normoandrogenemic PCOS.

\section{Prevalence of MetS and dyslipidemia}

While the overall rate of metabolic syndrome in women with PCOS was $22.5 \%$, within women diagnosed as PCOS according to the AES-PCOS criteria and normoandrogenemic PCOS, these rates were 25.4 and $10.3 \%$, respectively $(p=0.01$; Table 3$)$. The respective odds ratio for MetS in women with AES-PCOS was 2.95 [95\% CI, 1.21-7.21] when compared with subjects classified as normoandrogenemic PCOS. The distribution of the components of metabolic syndrome is also presented in Table 3. Regarding the lipid profile, the concentrations of HDLcholesterol, TG, TG/HDL-cholesterol ratio, and VLDL-C were significantly different in women with AES-PCOS from the subjects with normoandrogenemic PCOS $(p=0.004, p=0.03$, $\mathrm{p}=0.002$, and $\mathrm{p}=0.02$, respectively; Table 2). The prevalence of low HDL-cholesterol in the group under the AE-PCOS Society criteria was higher than that of normoandrogenemic PCOS (59.4 vs $41.2 \%$, respectively; $p=0.002$; Table 3 ), while the preva- lence of low HDL-cholesterol was $56.1 \%$ in the whole study group. While the prevalence of elevated total cholesterol $(\geq 200$ $\mathrm{mg} / \mathrm{dL})$ and LDL-C $(\geq 130 \mathrm{mg} / \mathrm{dL})$ parameters in the whole population were 18.3 and $14.6 \%$, respectively, there were no statistically significant differences between women diagnosed as PCOS according to the AE-PCOS Society criteria and women with normoandrogenemic PCOS ( $18.6 \%$ vs. $17.2 \%$ and $15.2 \%$ vs. $11.6 \% ; p=0.77$ and $p=0.39$, respectively). While the prevalence of elevated TG $(\geq 150 \mathrm{mg} / \mathrm{dL}$ ) was $21.5 \%$ in the whole study group, within the patients diagnosed as PCOS according to the AE-PCOS Society criteria and normoandrogenemic PCOS, these rates were 23.0 and $14.9 \%$, respectively $(p=0.09)$. While the prevalence of elevated TC to HDL-cholesterol ratio $(\geq 5.6)$ in the whole population was $7.6 \%$ within women diagnosed as PCOS according to AE-PCOS, this rate did not differ from subjects with normoandrogenemic PCOS (8.5 and 3.5\%, respectively; $\mathrm{p}=0.12$ ).

\section{Discussion}

In the current study, the prevalence of PCOS according to the Rotterdam and AE-PCOS Society criteria in a Caucasian population were 9.3 and $6.8 \%$, respectively. Although the results of the current study are in concurrence with the previously reported frequency's ranging from 11.2 to $20.9 \%$ according to the Rotterdam criteria $(18,26-28)$, we found lower figures than a prior Turkish cohort study using the AE-PCOS Society criteria (18). 
Further, data from this study illustrated that the prevalence of insulin resistance and impaired glucose tolerance barely rise in the overall PCOS population (31.7 and 17.8\%, respectively) when compared to that reported from another study of Turkish women with PCOS (29). This study has also shown that women with PCOS have a higher prevalence of metabolic syndrome (22.5\%) than that consistently reported from the literature, albeit with a varying rate $(17,18,28)$.

Although there is limited data on the association of the new phenotype (normoandrogenemic PCOS) and its implications on the metabolic profile, it has been reported that the normoandrogenemic PCOS phenotype has a milder metabolic syndrome with a rather similar rate of insulin resistance (30). The evidence currently suggests that patients with PCOS diagnosed according to AE-PCOS Society criteria have the most severe metabolic features $(15,17)$. Women in PCOS without hyperandrogenism are still the subject of debate $(2,15,16)$. The Androgen Excess and PCOS Society suggested that PCOS initially should be accepted as a condition of hyperandrogenism (15). The current study demonstrated that in women diagnosed according to AE-PCOS Society criteria, the proportion of increased serum glucose level was double in comparison with that in women identified as normoandrogenemic PCOS, while the rate of women with an abnormal glucose tolerance test and insulin resistance rose by almost $10 \%$. These findings appear to support the previous observations that women with normoandrogenemic PCOS exhibit a small proportion of hyperglycemia and insulin resistance $(31,32)$.

An impaired lipid profile is a prevalent finding in women with PCOS $(33,34)$, and also it was recently noted that the TG, LDL-cholesterol, and TC levels are significantly higher in obese PCOS women than in lean/normo-weight PCOS women, irrespective of the PCOS phenotypes (35). Women with hyperanderogenemic PCOS showed the most atherogenic lipid profiles, with higher apolipoprotein B compared to the other PCOS phenotypes (36). However, conflicting data are present in the literature. Moreover, despite the similar rate of IR in BMI-matched PCOS phenotypes, free testosterone and FAI were positively correlated with triglyceride as well as being inversely correlated with HDL-cholesterol (37). Conversely, other researchers speculate that lipid abnormalities are not different in women with hyperandrogenemic PCOS $(38,39)$. In accordance with the previous study, we also observed that women with normoandrogenemic PCOS have a lower triglyceride/HDL-cholesterol ratio, triglyceride, and VLDL-cholesterol concentrations as well as higher HDL-cholesterol levels when compared with those of women diagnosed as PCOS according to the AES-PCOS criteria, although BMI was not significantly different between the PCOS phenotypes (37). These findings suggest that hyperandrogenemia may adversely affect the lipid metabolism regardless of the presence of MetS or IR. However, the higher prevalence of decreased HDL-cholesterol with a similar rate of elevated TG, total cholesterol, and LDL-cholesterol observed in this study may indicate the effects of insulin resistance on HDLcholesterol. Further, this finding would be an indirect reflection of increased ovarian androgen secretion in women with PCOS, as proposed in previous reports $(40,41)$.
According to the new diagnostic criteria $(2,13)$, PCOS may de facto involve a large-scale number of abnormalities, consisting of women with mild clinical and hormonal abnormalities $(42,43)$. As regards metabolic syndrome (evaluated with the International Diabetes Federation criteria), our cohort study showed a proportion of $22.1 \%$ had it in the overall PCOS population according to the Rotterdam criteria consistent with a previous cohort study in a Mediterranean population (44). Furthermore, women diagnosed with PCOS according to the AE-PCOS Society criteria had a higher rate of MetS than those with normoandrogenemic PCOS (20.4 vs $9.2 \%$ ). This significant difference is in line with previous studies (45-48). The explanation for this quite pronounced difference may be that the key features of MetS comprising abdominal obesity, insulin resistance, and impaired glucose tolerance are present less frequently in normoandrogenemic PCOS (24). Indeed, a current meta-analysis showed that elevated serum androgen levels have a positive correlation with the prevalence of MetS (49). Overall, women with normal androgen levels appear to be part of the milder PCOS spectrum, but this might be due to the less frequently elevated blood glucose level, which is related with lower health-risk-related factors.

It has also been found that subclinical atherosclerosis expressed as low-grade inflammation (increased hs-CRP levels) could be a factor for an increased risk of cardiovascular disease and DM type 2 in women with PCOS (50-52). Moreover, an elevation in circulating hs-CRP irrespective of obesity is evidence that chronic low-grade inflammation may have an effect on the pathogenesis of PCOS, particularly in hyperanderogenemic phenotypes $(4,53)$. In accordance with previous reports, we also observed a significant elevation of hs-CRP in women with hyperanderogenemia compared to in women with normal androgen levels, despite there being no difference in BMI between the phenotypes $(4,53)$. This assumes that a high androgen level independently may be a factor that affects the establishment of cardiovascular disease in women diagnosed as PCOS.

Limitations for the study include that the methodology of study was a retrospective cohort, and also the numbers in the normoandrogenemic PCOS group were too small to assess the statistical significance for the oral glucose test intolerance.

In conclusion, women with hyperandrogenemic PCOS usually present an impaired lipid profile, insulin resistance, and abnormal glucose tolerance, which may be related to the subsequent development of MetS. PCOS patients with androgen excess appear to be exposed to a higher risk of cardiovascular disease than normoanderogenemic PCOS patients $(54,55)$. The observations of the present study suggest that the androgen excess per se is related with a long-term-health-risk of PCOS. This new data may be of reference in informing women diagnosed as PCOS, even though further prospective studies are needed to validate this proposition.

Ethics Committee Approval: Ethics committee approval was received for this study from the ethics committee of Inönü University.

Informed Consent: N/A.

Peer-review: Externally peer-reviewed. 
Author Contributions: Concept - E.Ç., I.T.; Design - E.Ç., I.T.; Supervision - B.B.; Materials - E.C., C..T.; Data Collection and/or Processing - S.E., P.K.; Analysis and/or Interpretation - E.Ç., B.A.; Literature Search - E.Ç., A.K.; Writing Manuscript - E.Ç., B.A.; Critical Review - B.B.

Conflict of Interest: No conflict of interest was declared by the authors.

Financial Disclosure: The authors declared that this study has received no financial support.

\section{References}

1. Koivunen R, Pouta A., Franks S, Martikainen H, Sovio U, Hartikainen AL, et al; Northern Finland Birth Cohort 1966 Study. Fecundability and spontaneous abortions in women with self-reported oligoamenorrhea and/or hirsutism: Northern Finland Birth Cohort 1966 Study. Hum Reprod 2008; 23: 2134-9.

2. Azziz R, Woods KS, Reyna R, Key TJ, Knochenhauer ES, Yildiz BO. The prevalence and features of the polycystic ovary syndrome in an unselected population. The Journal of clinical endocrinology and metabolism. 2004; 89: 2745-9.

3. Hull MG. Epidemiology of infertility and polycystic ovarian disease: endocrinological and demographic studies. Gynecological endocrinology: the official journal of the International Society of Gynecological Endocrinology. 1987; 1: 235-45.

4. Escobar-Morreale HF, Luque-Ramirez M, San Millan JL. The molecular-genetic basis of functional hyperandrogenism and the polycystic ovary syndrome. Endocr Rev 2005; 26: 251-82.

5. Rebar R, Judd HL, Yen SS, Rakoff J, Vandenberg G, Naftolin F. Characterization of the inappropriate gonadotropin secretion in polycystic ovary syndrome. J Clin Invest 1976; 57: 1320-9.

6. Amowitz LL, Sobel BE. Cardiovascular consequences of polycystic ovary syndrome. Endocrinology and metabolism clinics of North America. 1999; 28(2): 439-58, viii. Epub 1999/06/03.

7. Dokras A. Cardiovascular disease risk factors in polycystic ovary syndrome. Semin Reprod Med 2008; 26: 39-44.

8. Dahlgren E, Friberg LG, Johansson S, Lindstrom B, Oden A, Samsioe $\mathrm{G}$, et al. Endometrial carcinoma; ovarian dysfunction--a risk factor in young women. Eur J Obstet Gynecol Reprod Biol 1991; 41: 143-50.

9. Knochenhauer ES, Key TJ, Kahsar-Miller M, Waggoner W, Boots LR, Azziz R. Prevalence of the polycystic ovary syndrome in unselected black and white women of the southeastern United States: a prospective study. J Clin Endocrinol Metab 1998; 83: 3078-82.

10. Asuncion M, Calvo RM, San Millan JL, Sancho J, Avila S, EscobarMorreale HF. A prospective study of the prevalence of the polycystic ovary syndrome in unselected Caucasian women from Spain. J Clin Endocrinol Metab 2000; 85: 2434-8.

11. Diamanti-Kandarakis E, Kouli CR, Bergiele AT, Filandra FA, Tsianateli TC, Spina GG, et al. A survey of the polycystic ovary syndrome in the Greek island of Lesbos: hormonal and metabolic profile. J Clin Endocrinol Metab 1999; 84: 4006-11.

12. Michelmore KF, Balen AH, Dunger DB, Vessey MP. Polycystic ovaries and associated clinical and biochemical features in young women. Clinical endocrinology. 1999; 51: 779-86.

13. Revised 2003 consensus on diagnostic criteria and long-term health risks related to polycystic ovary syndrome (PCOS). Hum Reprod 2004; 19: 41-7.

14. Zawadzki JK DA. Diagnostic criteria for polycystic ovary syndrome: towards a rational approach. Polycystic Ovary Syndromes. In: Dunaif AGJ, Haseltine F (eds): Boston: Blackwell Scientific; 1992. p. 377-84.

15. Azziz R, Carmina E, Dewailly D, Diamanti-Kandarakis E, EscobarMorreale HF, Futterweit W, et al. Task Force on the Phenotype of the Polycystic Ovary Syndrome of The Androgen Excess and PCOS Society. The Androgen Excess and PCOS Society criteria for the polycystic ovary syndrome: the complete task force report. Fertil Steril 2009; 91: 456-88.

16. Revised 2003 consensus on diagnostic criteria and long-term health risks related to polycystic ovary syndrome. Fertil Steril 2004; 81: 1925.

17. Moran L, Teede H. Metabolic features of the reproductive phenotypes of polycystic ovary syndrome. Hum Reprod Update 2009; 15 : 477-88.

18. Yildiz BO, Bozdag G, Yapici Z, Esinler I, Yarali H. Prevalence, phenotype and cardiometabolic risk of polycystic ovary syndrome under different diagnostic criteria. Hum Reprod 2012; 27: 3067-73.

19. Moran LJ, Misso ML, Wild RA, Norman RJ. Impaired glucose tolerance, type 2 diabetes and metabolic syndrome in polycystic ovary syndrome: a systematic review and meta-analysis. Hum Reprod Update 2010; 16: 347-63.

20. Ferriman D, Gallwy JD. Clinical assessment of body hair growth in women. J Clin Endocrinol Metab 1961; 21: 1440-7.

21. Matthews DR, Hosker JP, Rudenski AS, Naylor BA, Treacher DF, Turner RC. Homeostasis Model Assessment - Insulin Resistance and Beta-Cell Function from Fasting Plasma-Glucose and Insulin Concentrations in Man. Diabetologia 1985; 28: 412-9.

22. Legro RS, Castracane VD, Kauffman RP. Detecting insulin resistance in polycystic ovary syndrome: purposes and pitfalls. Obstet Gynecol Surv 2004; 59: 141-54.

23. American Diabetes Association. Standards of Medical Care in Diabetes--2010. Diabetes Care 2010; 33: S11-61.

24. A worldwide consensus definition for the metabolic syndrome. Rev Panam Salud Publica 2005; 18: 451-4.

25. Talbert RL. Role of the National Cholesterol Education Program Adult Treatment Panel III guidelines in managing dyslipidemia. Am J Health-Syst Ph 2003; 60: S3-8.

26. Wiltgen D, Spritzer PM. Variation in metabolic and cardiovascular risk in women with different polycystic ovary syndrome phenotypes. Fertil Steril 2010; 94: 2493-6.

27. Boyle JA, Cunningham J, O'Dea K, Dunbar T, Norman RJ. Prevalence of polycystic ovary syndrome in a sample of Indigenous women in Darwin, Australia. Med J Aust 2012; 196: 62-6.

28. March WA, Moore VM, Willson KJ, Phillips DIW, Norman RJ, Davies MJ. Prevalence of the Polycystic Ovary Syndrome in a Community Sample Assessed under Contrasting Diagnostic Criteria. Aust Nz J Obstet Gyn 2010; 50: 16-7.

29. Moran C, Tena G, Moran S, Ruiz P, Reyna R, Duque X. Prevalence of Polycystic Ovary Syndrome and Related Disorders in Mexican Women. Gynecol Obstet Inves 2010; 69: 274-80.

30. Celik C, Abali R, Bastu E, Tasdemir N, Tasdemir UG, Gul A. Assessment of impaired glucose tolerance prevalence with hemoglobin A1c and oral glucose tolerance test in 252 Turkish women with polycystic ovary syndrome: a prospective, controlled study. Hum Reprod 2013; 28: 1062-8.

31. Panidis D, Tziomalos K, Misichronis G, Papadakis E, Betsas G, Katsikis I, et al. Insulin resistance and endocrine characteristics of the different phenotypes of polycystic ovary syndrome: a prospective study. Hum Reprod 2012; 27: 541-9.

32. Broekmans FJ, Knauff EA, Valkenburg O, Laven JS, Eijkemans MJ, Fauser BC. PCOS according to the Rotterdam consensus criteria: Change in prevalence among WHO-II anovulation and association with metabolic factors. BJOG 2006; 113: 1210-7.

33. Guastella E, Longo RA, Carmina E. Clinical and endocrine characteristics of the main polycystic ovary syndrome phenotypes. Fertil Steril 2010; 94: 2197-201. 
34. Legro RS, Kunselman AR, Dunaif A. Prevalence and predictors of dyslipidemia in women with polycystic ovary syndrome. Am J Med 2001; 111: 607-13.

35. Cussons AJ, Stuckey BG, Watts GF. Cardiovascular disease in the polycystic ovary syndrome: new insights and perspectives. Atherosclerosis 2006; 185: 227-39.

36. Rizzo M, Berneis K, Hersberger M, Pepe I, Di Fede G, Rini GB, et al. Milder forms of atherogenic dyslipidemia in ovulatory versus anovulatory polycystic ovary syndrome phenotype. Hum Reprod 2009; 24: 2286-92.

37. Castelo-Branco C, Steinvarcel F, Osorio A, Ros C, Balasch J. Atherogenic metabolic profile in PCOS patients: role of obesity and hyperandrogenism. Gynecological endocrinology: the official journal of the International Society of Gynecol Endocrinol 2010; 26: 736-42.

38. Maffei L, Murata Y, Rochira V, Tubert G, Aranda C, Vazquez M, et al. Dysmetabolic syndrome in a man with a novel mutation of the aromatase gene: effects of testosterone, alendronate, and estradiol treatment. J Clin Endocrinol Metab 2004; 89: 61-70.

39. Carmina E, Chu MC, Longo RA, Rini GB, Lobo RA. Phenotypic variation in hyperandrogenic women influences the findings of abnormal metabolic and cardiovascular risk parameters. J Clin Endocrinol Metab 2005; 90: 2545-9.

40. Carmina E, Lobo RA. Prevalence and metabolic characteristics of adrenal androgen excess in hyperandrogenic women with different phenotypes. J Endocrinol Invest 2007; 30: 111-6.

41. Rizzo M, Longo RA, Guastella E, Rini GB, Carmina E. Assessing cardiovascular risk in Mediterranean women with polycystic ovary syndrome. J Endocrinol Invest 2011; 34: 422-6.

42. Karkanaki A, Piouka A, Katsikis I, Farmakiotis D, Macut D, Panidis D. Adiponectin levels reflect the different phenotypes of polycystic ovary syndrome: study in normal weight, normoinsulinemic patients. Fertil Steril 2009; 92: 2078-81.

43. Carmina E, Orio F, Palomba S, Longo RA, Cascella T, Colao A, et al. Endothelial dysfunction in PCOS: role of obesity and adipose hormones. Am J Med 2006; 119: 356.e1-6.

44. Barber TM, Wass JA, McCarthy MI, Franks S. Metabolic characteristics of women with polycystic ovaries and oligo-amenorrhoea but normal androgen levels: implications for the management of polycystic ovary syndrome. Clin Endocrinol 2007; 66: 513-7.

45. Amato MC, Galluzzo A, Finocchiaro S, Criscimanna A, Giordano C. The evaluation of metabolic parameters and insulin sensitivity for a more robust diagnosis of the polycystic ovary syndrome. Clin Endocrinol 2008; 69: 52-60.

46. Apridonidze T, Essah PA, Iuorno MJ, Nestler JE. Prevalence and characteristics of the metabolic syndrome in women with polycystic ovary syndrome. J Clin Endocrinol Metab 2005; 90: 1929-35.

47. Cussons AJ, Watts GF, Burke V, Shaw JE, Zimmet PZ, Stuckey BG. Cardiometabolic risk in polycystic ovary syndrome: a comparison of different approaches to defining the metabolic syndrome. Hum Reprod 2008; 23: 2352-8.

48. Cheang KI, Huszar JM, Best AM, Sharma S, Essah PA, Nestler JE. Long-term effect of metformin on metabolic parameters in the polycystic ovary syndrome. Diab Vasc Dis Res 2009; 6: 110-9.

49. Tziomalos K, Katsikis I, Papadakis E, Kandaraki EA, Macut D, Panidis D. Comparison of markers of insulin resistance and circulating androgens between women with polycystic ovary syndrome and women with metabolic syndrome. Hum Reprod 2013; 28: 785-93.

50. Brand JS, van der Tweel I, Grobbee DE, Emmelot-Vonk MH, van der Schouw YT. Testosterone, sex hormone-binding globulin and the metabolic syndrome: a systematic review and meta-analysis of observational studies. Int J Epidemiol 2011; 40: 189-207.

51. Türkçüoğlu I, Kafkasli A, Meydanli MM, Ozyalin F, Taşkapan C. Independent predictors of cardiovascular risk in polycystic ovarian syndrome. Gynecol Endocrinol 2011; 27: 915-9.

52. Karaer A, Cavkaytar S, Mert I, Buyukkagnici U, Batioglu S. Cardiovascular risk factors in polycystic ovary syndrome. J Obstet Gynaecol 2010; 30: 387-92.

53. Athyros VG, Kakafika AI, Karagiannis A, Mikhailidis DP. Do we need to consider inflammatory markers when we treat atherosclerotic disease? Atherosclerosis 2008; 200: 1-12.

54. Daskalopoulos GN, Karkanaki A, Karagiannis A, Mikhailidis DP, Athyros VG. Is the risk for cardiovascular disease increased in all phenotypes of the polycystic ovary syndrome? Angiology 2011; 62: 285-90.

55. Wild RA, Carmina E, Diamanti-Kandarakis E, Dokras A, EscobarMorreale HF, Futterweit W, et al. Assessment of cardiovascular risk and prevention of cardiovascular disease in women with the polycystic ovary syndrome: a consensus statement by the Androgen Excess and Polycystic Ovary Syndrome (AE-PCOS) Society. J Clin Endocrinol Metab 2010; 95: 2038-49.

56. Makedos A, Goulis DG, Papanikolaou A, Panidis D. Serum highsensitivity C-reactive protein and homocysteine changes during hormonal therapy in women with polycystic ovary syndrome: a prospective, matched study. Angiology 2010; 61: 595-601. 